\title{
Web-Based Problem-Solving System for Learner Motivation of Engineering Students
}

\author{
Ehsan Ahmed, Musfiq Rahman, and Hafizur Rahman \\ Thompson Rivers University, Kamloops, BC \\ Corresponding Author Email Address: eahmed@tru.ca
}

\begin{abstract}
This paper addresses the construction of a novel approach to enhance learner motivation as it relates to the learning process. The foundational principles of motivation are discussed. The impact of instructional technology on student motivation through an innovative web-based problem-solving system is the main focus of this study. The development of the web-based problem-solving system and its evaluation process are considered for addressing the foundational principles of motivation. The proposed system is customizable to provide feedback to both students and instructors. The outcome of this research will help establish a suitable instructional design model of motivation for engineering students. The process also has prospects for applications in other disciplines.
\end{abstract}

Keywords: Motivation, Web-based learning, Engineering, Learning outcomes.

\section{INTRODUCTION}

Motivation to learn is paramount to student success and is considered as the principal driving force of learning. However, there is no guaranteed way to motivate a person to study, to learn or to work. In intrinsic motivation, the individual engages in work for the pleasure of manipulating objects or ideas or for solving a problem. The need to explore will manifest itself in the desire to read, study and learn about scientific and intellectual advances. The sources of motivation are complex. Motivational planning can be integrated with instructional planning, or it can be used in addition to instructional planning. Such planning can help avoid a serious pitfall common to teaching: blaming the learners for being unresponsive to instruction.

The effects of using technology on student's motivation and engagement in classroom-based learning have been actively researched. Appropriate use of technology can enhance regular classroom education by motivating students [6][7]. It also has the benefit of enriching the learning environment for students and educators alike [4]. Research indicates [5][6] that to create an effective learning environment a modern teacher should factor in the positive impact of technology and student's motivation. Research also shows that students are positively motivated through the use of appropriate technology in the classroom. While many teachers feel that technology is helpful but not necessary, contemporary students frequently view technology as a central component of active learning [11]. Traditionally, the measurement of motivation has been conducted either through an assessment of time that students freely spent on a problem [10] or by using tools such as surveys and interviews. The former can be difficult to keep track of and the latter has its limitations in terms of additional time and expense required.

This paper presents a web-based e-learning problemsolving platform for an Engineering Fluid Mechanics course. Both the pipe flow and open channel flow problems are considered in formulating the system. Our system follows the learning levels outlined in Bloom's Taxonomy [2], providing feedback for each level. The system is flexible enough to allow students to choose options at various stages of problem-solving. It also provides intermediate results that help confirm the calculations along the way, which is useful in identifying faults in a problem-solving approach and helps enhance learners' motivation. Pre-stored examples in the system with intermediate results will assist the student to understand the relevant theory and further develop engineering intuition.

This paper also discusses the factors related to the development process that influence the motivation to learn based on the foundational principles of motivation. The inbuilt process within the proposed software can facilitate the measurement of motivation in various ways. The novelty of our system is that it allows flexibility for customization by the instructor, and its ability to provide feedback to both students and instructors.

\section{FOUNDATIONAL PRINCIPLES OF MOTIVATION}

There are four foundational principles that motivate students to learn Inclusion, Attitude, Meaning and Competence [13].

\subsection{Inclusion}

Inclusion is the awareness of learners that they are part of an environment in which they and their instructor are mutually respected and connected. This awareness creates a favorable social climate for learning. It can be established 
by ensuring that the environment is free from fear, threat, or humiliation. Shared understanding among group members that support each other's well-being is also necessary to develop inclusion and it can be instilled in the first class during the introduction of the course. The student should know the expectations of their instructor.

An important aspect of inclusion is to ensure that all learners have equal access to learning resources. With the proposed web-based learning systems, all learners will have access to resources from anywhere at no cost to them. This makes it a public good as it satisfies both the nonrivalry and non-exclusion principles of public goods.

\subsection{Attitude}

Attitude combines concepts, information, and emotions that help people make sense of their world. It suggests behavior that is most useful in dealing with the world. It can be developed by creating a favorable disposition toward the learning experience through personal relevance and choice. Essential components of building a positive attitude include allowing time for learners' self-reflection and for cultivating relationships between learner and instructor.

Our conception of web-based systems is intended to help solidify concepts, improve the informational efficiency of the learning process, and progress of learning to create positive emotions towards learning.

\subsection{Meaning}

Meaning sustains learner involvement. Meaning can be achieved by creating challenging and thoughtful learning experiences that include learners' perspectives and values. The web-based system can be easily configured to meet these objectives. Students will get instant feedback at three levels of detail and will have the opportunity to work on progressively challenging problems at the pace they individually determine for themselves.

\subsection{Competence}

Competence builds learner confidence. It can be achieved by creating an understanding that learners are effective in learning something they value. This can be developed throughout the course by providing the opportunity to solve problems with an increasing level of complexity and difficulty.

The web-based system is designed to provide opportunities for the progressively increased active involvement of the learners to make progress towards higher levels of learning (e.g. as defined by Bloom's [2]).

In all, the proposed web-based problem-solving system will have a positive impact on students' learning, and it will enhance learner motivation specifically in terms of meaning and competence.

\section{DEVELOPMENT OF WEB BASED PROBLEM-SOLVING SYSTEM}

In this research, a web-based problem-solving system for fundamental fluid mechanics course of engineering program is proposed. At present, in the third-year fluid mechanics course in Architectural \& Engineering Technology (ARET) program of Thompson Rivers University (TRU), the basic formulation of various problems such as pipe flow, pump \& pressure, pipe network distribution, open channel flow related problems are solved either using traditional hand calculation method or by using a scientific programmable calculator. Hand calculation method using formula and calculator takes considerable time and limits the learner's ability to explore all the learning objectives of the course. Although this method directly involves the learners and helps develop their cognitive thinking, it may distract the learners in achieving the course objectives and outcomes because students have to spend much time calculating details rather than focusing on the actual big picture of the problem.

When using a programmable calculator, the students only get the final results by inserting the inputs associated with the program. In this case, most of the learning sequences are performed by the inbuilt ability of these powerful calculators. Therefore, students using these types of calculators are liable to be lacking in engineering common sense and intuition. This lack of engineering common sense may not be apparent as most engineering solutions are based on existing solutions and common patterns. However, when innovative new solutions are needed, this lack of intuition may lead to potentially catastrophic mistakes.

Although there are some web-based free to use equation solvers available on the web [8], they often do not have options for customizing the equations and options for revealing the intermediate steps in a calculation. Furthermore, they do not provide feedback on the student's motivation and learning level to the instructor. In fact, for these tools, the instructor cannot even know if a student used them or not for solving the equation. Thus, there is a need to develop a problem-solving system that will not only enhance student's motivation towards learning, but also improve the engineering common sense and intuition. Therefore, we propose a web-based system that will have the following features.

The web-based program targets each of the levels of the sequences outlined in Bloom's [2] separately, rather than as an integrated tool. Choosing options at various stages of problem-solving should be left to the learner rather than to have a predetermined and rigid built-in system. The program should provide intermediate results that can help confirm the calculations along the way. These intermediate results can be useful in identifying faults in a problemsolving approach and help enhance learners' motivation. Pre-stored examples in the system with intermediate results 
will help the student to understand the relevant theory and help develop engineering intuition.

Studies have shown that instructional technology, when applied appropriately, makes a positive impact on student motivation, academic outcomes and skills [5][6][7]. The proposed web-based problem-solving system is one such technology.

The use of computer software for teaching has many advantages [1][3][12]. Exposure to computers as the problem-solving tool in engineering practice reduces the time for problem-solving and gives flexibility in terms of availability of resources. Besides, a graphical representation of the details of a problem and repetitive use with less effort can be significant advantages towards learners' motivation.

\section{DESIGN OF THE SOLVING SYSTEM}

In this section, we introduce a web-based problemsolving system for the engineering course mentioned earlier. The developed web system supports personalized learning through flexible customization of the contents based on learning objectives. The framework provides a web-based interface through which engineering students can perform lengthy calculations and are able to inspect intermediate results to check and solidify their learning in the fluid mechanics course. Although the proposed framework is designed for engineering students in mind, it can easily be extended to use in other disciplines, such as biology, statistics, economics and other disciplines in science.

In microeconomics, for example, a system of equations for markets for demand and supply may be used to examine impacts of policy changes such as taxes and subsidies on market prices, quantities exchanged and tax revenues under various assumptions about demand and supply conditions. In macroeconomics, a system of equations defining a macro model can be used to examine impacts of fiscal and monetary policy changes or changes in trade agreements, among many other possibilities.

The application framework is composed of two major modules: setup and execution modules.

\subsection{Setup Module}

In this web-based module, the instructors select and set up the formulas that the students will solve in a course. Instructors can also define new formulas and their related characteristics at this stage. Engineering formulas contain several variables with some unknown values that need to be calculated by solving the formulae. For example, the steady flow energy equation as shown in Eq.1 of fluid mechanics have several variables such as pressure $(p)$, velocity (v), elevation (z), and shaft work $\left(w^{\prime}\right)$. Furthermore, the formula of friction head loss $\left(h_{f}\right)$ also have some more variables. In this situation, a program that solves the energy equation for up to two pipes in series requires a solver that will solve the equation.

CEEA-ACEG20; Paper 001

Concordia and McGill Universities; June 18 - 21, 2020- 3 of 6 -

$$
\frac{p_{1}}{\rho g}+\frac{v_{1}^{2}}{2 g}+z_{1}-h_{f}=\frac{p_{2}}{\rho g}+\frac{v_{2}^{2}}{2 g}+z_{2}+\frac{w^{\prime}}{g} \quad \text { Eq. } 1
$$

The instructors can also customize a formula and choose some variables to remain known, and other variables to remain unknown. The objective would be to calculate the unknown values by solving the equation by assigning values to the known variables. Once the formulae are selected, the setup is complete, and the selected formulae will be displayed to the students in the execution module. A use case diagram for this module is shown in Fig. 1.

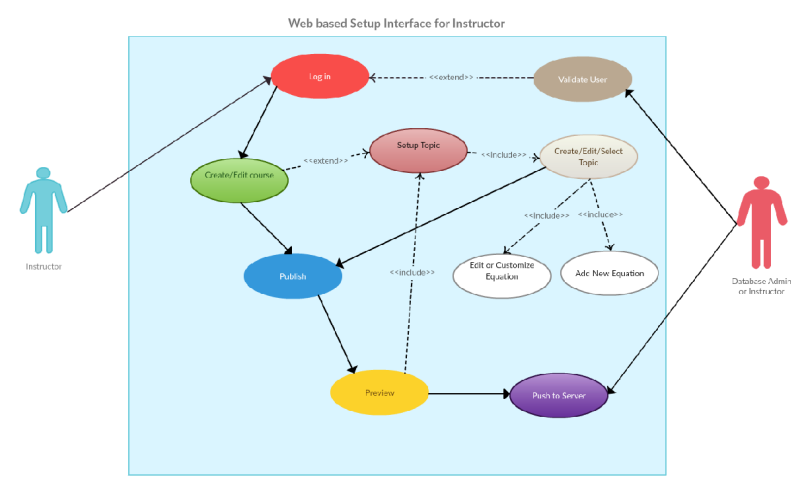

Fig. 1. Shows the use case diagram for the setup module of the system.

\subsection{Execution Module}

This is a web-based module for the student. After successful login, they will be able to view the selected formulae for their course. The formulae will be shown with some known and unknown variables as customized by the instructor. Students will have to enter valid values for the known variables in the equation to solve it for the unknown values. The system shows the full detail steps of the calculation for the equation, the intermediate results, and the final result. For example, depending on the motivation level, a student may want to solve the equation by hand and want to verify his/her final result using our web-based application. In other cases, students may need to know if they are on the right track in solving the equations by looking at the intermediate steps. With this design concept, our system will support and accommodate different student's motivations as outlined in the foundational principles of motivation [13]. We plan to add a novel feature where the students' activities will be tracked and automatic feedback will be provided to the instructor. Fig.2 shows the use-case diagram of the execution module of our system.

We have already conducted the requirement analysis of our proposed system and selected front-end and back-end 


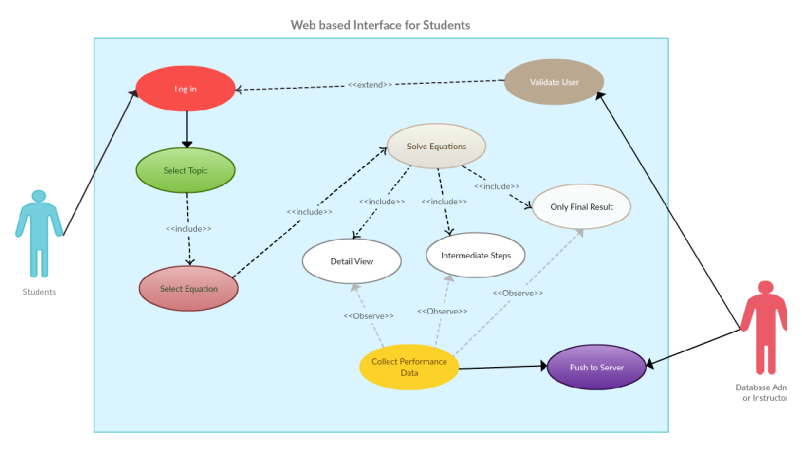

Fig.2. Shows the use case diagram for the execution module of the system

technologies for developing the application. One of the critical requirements of our system design is that the instructors would require a flexible method to define new equations with many variables. These equations may contain various mathematical operators and symbols for advanced operations. The system should be able to recognize different variables, symbols, and constants as operators, and then perform different operations to calculate the unknown values. To handle this requirement, we have designed a module that converts the equations from their natural representation to a JSON formatted object [9]. We then store the objects into a server for accessing them from anywhere. Furthermore, for each equation, we created a solver module that is associated with the equation. When a student selects the solve button of the execution interface, the system invokes the associated solver to solve the equation and present the detail, intermediate, and/or final results. For the front and backend development, we used HTML with JavaScript, and PHP with MySQL respectively.

\subsection{The Designed System}

In our web-based system, there are six tabs namely, PFLO, PPIPE, OPEN Channel Flow, Specific Energy, Hydraulic Jump and RFLO located at the top as dropdown menu and four tabs in the side navigation bar (see Fig.3). The top six tabs are used to solve problems related to the fluid mechanics course whereas the side navigation tabs provide solved examples and links to the chapter of the text. Instructors can define new tabs with new formulae and their related characteristics. Each tab contains a collection of various solving tools that solve variables associated with the equations in a tab.

In fluid mechanics, if all the elements of a pipe system are connected in a series, the system is known as a pipeline. The 'PFLO' program is designed to solve pipeline problems with two different diameters of pipes. This program can be used to solve pipeline problems involving up to four different diameters of pipes on an iterative basis.

CEEA-ACEG20; Paper 001

Concordia and McGill Universities; June 18 - 21, 2020- 4 of 6 -

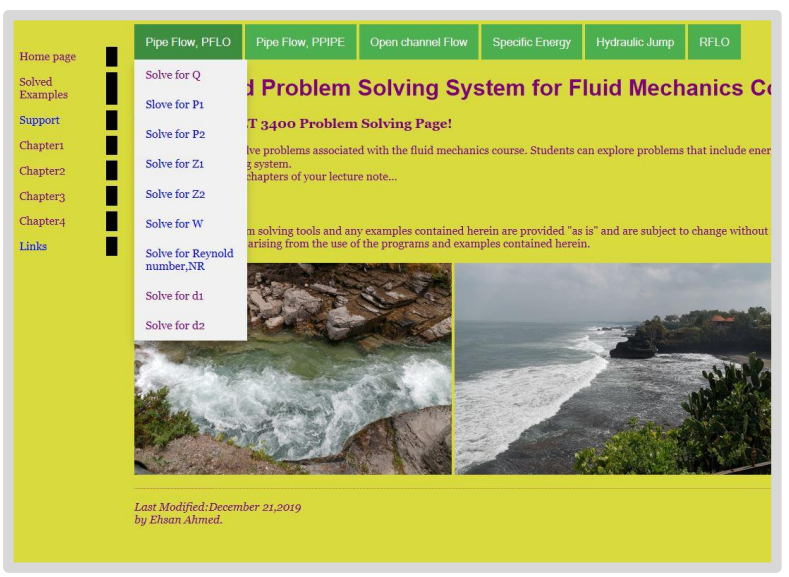

Fig. 3. Home page of the problem-solving system

A pipe network is a system in which the elements are not in series. One of the simplest forms of pipe networks consists of a single branch flow as shown in Fig. 4.

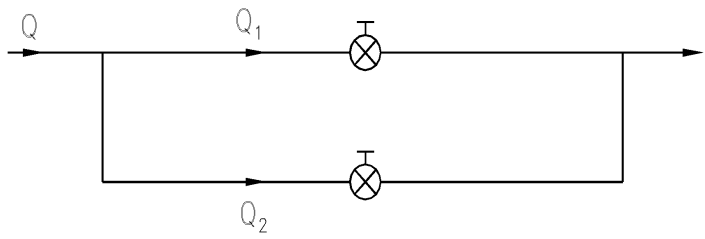

Fig.4. Simple pipe network system

The solution of such a system is based on two principles. The first principle is that continuity or conservation of mass can be applied to each node. In other words, the sum of the flows entering a node must equal the sum of the flows leaving the node. In Fig.4, this principle yields the following equation:

$$
Q=Q_{1}+Q_{2}
$$

The second principle states that the energy head at any given point can only have a single unique value. This implies that the head loss to friction through branch 1 must be the same as the head loss through branch 2,

$$
h_{f 1}=h_{f 2}
$$

The program 'PPIPE' solves Eq.3 for the simple case of a single branch flow. The program only solves for $\mathrm{Q}_{1}$. $\mathrm{Q}_{2}$ is then obtained from Eq.2.

In Open Channel Flow tab, the programs are designed to solve Manning's equation. The Program 'TRAP' will solve for trapezoidal, rectangular and vee-shaped channels. This program can be used to solve Manning's equation for any of the variables. Program ' $Q$ ' under the 'Open Channel Flow' tab solves Manning's equation for a circular section. In open-channel flow, gravity forces predominate and we define the Froude number, $N_{F}$, as being the ratio of the inertia force to the gravity force in the flow. The program ' $\mathrm{Fr}^{\prime}$ inside the Open Channel Flow tab can be used to 
determine the Froude number for a specified discharge in a rectangular open channel.

The Specific Energy in an open-channel is defined as the energy head measured with respect to the channel bottom at the section under consideration. For a rectangular channel $(A=b d)$, the equation for specific energy becomes:

$$
E=\frac{Q^{2}}{b^{2} d^{2} 2 g}+d
$$

The program inside the Specific Energy Tab 'SE' is designed considering the above equation.

A hydraulic jump is a natural phenomenon in openchannel flow that occurs when supercritical flow is undergoing a rapid deceleration. It is an abrupt reduction in flow velocity with a sudden increase in water depth in the downstream direction. For a rectangular channel, the depth, $d_{2}$, after the jump can be calculated from momentum considerations if the depth before the jump, $d_{l}$, and the flow rate, $Q$, are known. The derivation assumes that the jump takes place in a horizontal channel, but reasonable results are obtained for the small slopes normally encountered in most engineering problems. The resulting equation is the following.

$$
d_{2}=-\frac{d_{1}}{2}+\left(\frac{2 Q^{2}}{b^{2} d_{1} g}+\frac{d_{1}^{2}}{4}\right)^{1 / 2}
$$

The program 'JUMP' under the 'Hydraulic Jump' tab is based on Eq.5.

The 'RFLO' program has been designed to solve problem related to the gradually varied flow. In gradually varied flow, the depth varies gradually along the length of the channel. Only steady flow (constant Q) is considered. The equation that describes how the depth varies along the channel length is obtained by differentiating the expression for the total head with respect to $x$, where $x$ is the distance along the channel. The equation thus obtained is a differential equation. It can be represented as:

$$
\frac{d d}{d x}=S_{0} \frac{1-\left(d_{n} / d\right)^{N}}{1-\left(d_{c} / d\right)^{M}}
$$

Eq. 6 can be integrated to determine the length for a given change in depth:

$$
\begin{aligned}
& \int_{0}^{L} d x=L \\
& =\int_{d 1}^{d 2} \frac{1}{S_{0} \frac{1-\left(d_{n} / d\right)^{N}}{1-\left(d_{c} / d\right)^{M}}} d d
\end{aligned}
$$

The program 'RFLO' is based on Eq.7, with $M$ and $N$ determined for rectangular channels. This program integrates between two different depths, $d_{1}$ and $d_{2}$, and gives the resulting length $L$ between the two depths. The programs 'TRAP' and 'DCRIT' within this tab must be run prior to executing the 'RFLO' program to set up the variables $d_{n}$ (normal depth) and $d_{c}$ (critical depth).

Students can navigate to different problem-solving pages by clicking on any solving tab as shown in Fig.4. The page shows the brief description of the topic, related equations, images and the fields for input parameters as depicted in Fig.5. Once the students enter all the known parameters and click on the "calculate" button, the system calculates the unknown variables and displays the results.

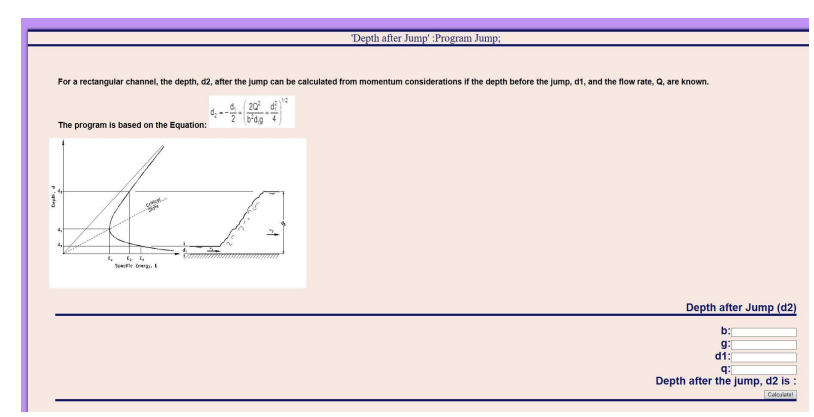

Fig. 5. Typical page for the calculation of the depth after the Hydraulic Jump

\section{BENEFITS OF THE SYSTEM}

Our platform will motivate students' learning by providing them with free and easy access tool they can use from anywhere to better understand complex engineering theories. Moreover, our system will provide feedback to the instructor on how much time a student spent on solving an equation. Additionally, it will send reports to the instructor on student's performance. This will help the instructor to track student's level of engagement and learning. The proposed features can be further used to conduct research on student motivation and can be customized for application in other disciplines.

\section{EVALUATION OF THE SYSTEM}

The evaluation of the web-based problem-solving system will be carried out by using it in a third-year fluid mechanics course. The first half of the course (before the mid-term) will use the traditional method for solving problems and the second half of the course will utilize the web-based problem-solving system. Then, we will monitor the students' performance and engineering intuition in light of the four foundational principles of motivations. The web-based problem-solving system will provide many prospects for evaluation of student learning efforts and the effectiveness of learning. First, the system will generate detailed data for the instructor on the use of the tool by individual students. The data will help the instructor 
identify struggling students and help better support for those students.

Second, statistical analysis of raw data would provide useful information to gauge the extent of student interest and use of the system. This information can be useful for developing strategies to further increase the participation of future students. Finally, data analysis combining the extent of the use of this learning tool and student performance (assignments, tests, exams and final grades) could also provide useful statistics on the effectiveness of the use of such tools for student learning outcomes.

\section{CONCLUSION}

This paper presents an innovative web-based problemsolving system related to a fundamental course in engineering. The system will help assess the impact on learners' urge to learn based on the four foundational principles of motivation. Years of teaching experience and review of relevant studies indicate that the designed system will enhance student motivation in terms of the foundational principles outlined in this paper. Our webbased learning system has potential applications in many other disciplines. The novelty of our method includes flexibility for customization by the instructor and its ability to provide feedback to both students and instructors.

\section{References}

[1] Ehsan Ahmed and Musfiq Rahman. 2016. Impact of Software Usage on Fundamental Engineering Courses. In Proceedings of the 21st Western Canadian Conference on Computing Education (WCCCE '16). ACM, New York, NY, USA, Article 23, 3 pages. https://doi.org/10.1145/2910925.2910942

[2] B. S. Bloom, M. B. Engelhart, E. J. Furst,W. H. Hill, and D. R. Krathwohl. 1956. Taxonomy of educational objectives. The classification of educational goals. Handbook 1: Cognitive domain. Longmans Green, New York.

[3] Anna Consortini. 2013. Advantages and disadvantages of using computers in education and research, In ETOP 2013 Proceedings. ETOP 2013 Proceedings, EThB1. http://www.osapublishing.org/abstract.cfm?URI=ETOP -2013-EThB1

[4] Keith Courville. 2011. Technology and Its Use in Education: Present Roles and Future Prospects. In Recovery School District Technology Summit. 19.

[5] James Francis. 2017. The Effects of Technology On Student Motivation And Engagement In ClassroomBased Learning. Ph.D. Dissertation. University of New England.

[6] Tina Heafner. 2004. Using Technology to Motivate Students to Learn Social Studies. Contemporary Issues in Technology and Teacher Education (CITE Journal),4, 1 (2004), 42-53.
[7] Brian C. Housand and Angela M. Housand. [n. d.]. The role of technology in gifted students' motivation. Psychology in the Schools 49, 7 ([n. d.]), 706-715. https://doi.org/10.1002/pits.21629arXiv:https://onlinelib rary.wiley.com/doi/pdf/10.1002/pits.21629

[8] Jabacus 2009. Jabacus Online Calculations. Retrieved Jan 30, 2020 from http://www.jabacus.com/index.php

[9] JSON [n. d.]. JSON: JavaScript Object Notation. Retrieved Mar 10, 2019 from https://www.json.org/

[10] Elizabeth L. Leo and David Galloway. 1996. Evaluating Research on Motivation: Generating More Heat Than Light? Evaluation \& Research in Education 10, 1(1996), 35-48. https://doi.org/10.1080/09500799608666904

[11] Kevin Thomas, Blanche O'Bannon, and Natalie Bolton. 2013. Cell Phones in the Classroom: Teachers Perspectives of Inclusion, Benefits, and Barriers. Computers in the Schools 30 (10 2013), 295-308. https://doi.org/10.1080/07380569.2013.844637

[12] Louis H. Turcotte and Howard B. Wilson. 1997. Computer Applications in Mechanics of Materials Using MATLAB. Prentice Hall PTR, Upper Saddle River, NJ, USA.

[13] Raymond J. Wlodkowski. 2017. Enhancing Adult Motivation to Learn A Comprehensive Guide for Teaching All Adults. John Wiley Sons, Incorporated. 